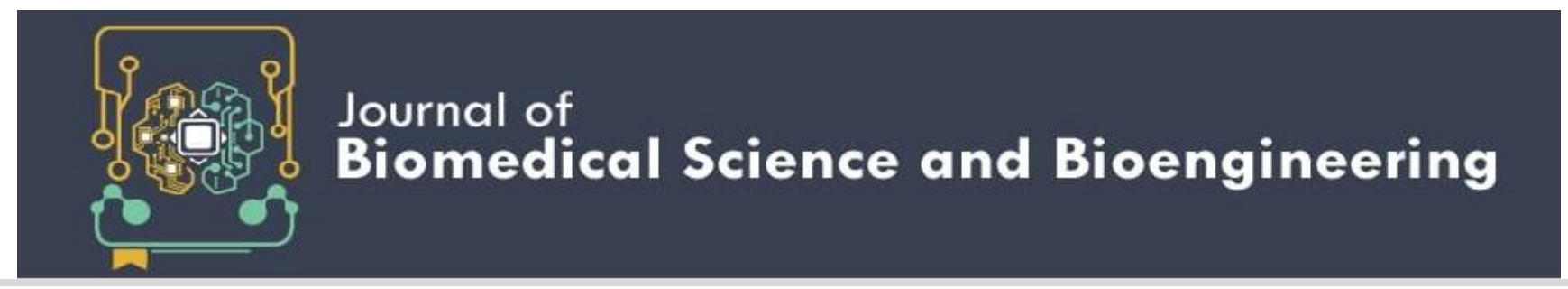

\title{
Multiparameter Biosignal Analysis in Elderly Ischemic Stroke Patients
}

\author{
Muhammad Zakariyah ${ }^{1}$, Alvin Sahroni2*, and Erlina Marfianti ${ }^{3}$ \\ ${ }^{I}$ Department of Informatics, Faculty of Industrial Technology, Universitas Islam Indonesia, Yogyakarta, Indonesia \\ ${ }^{2}$ Deparment of Electrical Engineering, Faculty of Industrial Technology, Universitas Islam Indonesia, Yogyakarta, Indonesia \\ ${ }^{2}$ Department of Medical Education, Medical Faculty, Universitas Islam Indonesia, Yogyakarta, Indonesia \\ *Corresponding Author: alvinsahroni@uii.ac.id
}

\begin{abstract}
Biosignal can provide information about body conditions, including physiological conditions of ischemic stroke. The regulation of blood in the brain is regulated through the mechanism of Cerebral Autoregulation (CA). Some parameters that can be used to determine this mechanism are Blood Flow Velocity (BFV) and Blood Pressure (BP). Stroke is also related to nervous system activity, which is represented through the Heart Rate Variability (HRV). This study aims to determine the relationship between those biosignals and their effects on the physiology of ischemic stroke sufferers. The subjects were divided into two groups (20 strokes and 20 controls). BFV data is obtained in the Middle Cerebral Artery (MCA), BP is obtained through the arteries of the upper arms, and 3 leads electrocardiogram is placed in the chest. The results showed that there was a relationship between BP and BFV in the control group ( $\mathrm{p}$-value $<0.05 ; \mathrm{r}=-0.574)$. This correlation was not found in the stroke group. The relationship between BP and HRV was only found in the stroke group, which was associated with high sympathetic activity and lower parasympathetic activity (p-values $<0.05$ and $r>0.4$ ). It was based on SDRR, RMSSD, CVRR, LF, and SD1 parameters. In the control group, there was no relationship between HRV and BP. The relationship between BFV and HRV in the control group was not found statistically. Still, in the stroke group, this relationship was found in the LF and LF/HF Ratio parameters (p-value $<0.05 ; \mathrm{r}>0.4$ ). Based on this research, parameters on HRV that can be used to determine the characteristics of stroke patients in all positions are MeanRR, VLF, and LF.
\end{abstract}

Keywords: Ischemic Stroke, Biosignal, Blood Pressure, Blood Flow Velocity, Heart Rate Variability

Article History: Received 4 July 2020; Accepted 20 March 2021; Available online: 29 March 2021

DOI: $10.14710 /$ jbiomes.2021.v1i1.7-16

\section{INTRODUCTION}

Blood is pumped by the heart to all parts of the body through blood vessels. Blood flows have different pressure and velocity, depending on where the blood vessels are located. In capillaries, blood flows a thousand times slower compare to the aorta [1].

Abnormal blood pressure causes serious medical conditions. Stroke is a disease closely related to excessive blood pressure (hypertension). Stroke occurs when blood flows into the brain is disrupted, which then damages brain cells. The effect of stroke depends on which brain part was damaged and also amount of damage it causes.

The main causes of stroke are ischemic (blockage) and hemorrhagic (bleeding). Ischemic strokes are more common than hemorrhagic strokes, with a prevalence rate of up to $85 \%$ of total stroke cases [2].

Ischemic stroke in elderly patients is a major public health problem, as its strong association with many medical complications, worse functional outcomes, and large health care costs. Compared with young ischemic stroke sufferers, elderly ischemic stroke sufferers show more adverse outcomes [3]. Young stroke patients also shown better neurological and functional recovery after stroke [4] [5].

In addition, young stroke patients also make it possible to have a better long-term survival rate. A post-stroke study of 3864 young stroke patients conducted by [6] found that mortality in the young group was $19 \%$ lower compared to elderly stroke patients. High mortality rates and lower outcomes in elderly ischemic stroke sufferers requires further study.
Changes in Heart Rate (HR), both tachycardia (rapid heart rate) and bradycardia (slow heart rate) is a phenomenon that is often found in acute stroke conditions. However, both cannot be used as benchmarks to determine the clinical condition in stroke patients [7]. Other parameters are needed to explain the condition of stroke patients, so it is possible to determine the medical treatment to be performed.

Post stroke, cerebral autoregulation becomes disturbed [8] [9]. Cerebral Autoregulation (CA) is the ability of blood vessels to maintain Cerebral Blood Flow (CBF) relatively constant to changes in blood pressure through myogenic, neurogenic, and complex metabolic mechanisms. $\mathrm{CBF}$ depends on the condition of blood vessels and arterial blood pressure. Blood pressure rises more than $75 \%$ in people who have an acute stroke [10]. The disruption of CA in stroke patients also causes wider atrophy in the brain region, as well as reduced functional ability of the body [11].

One mechanism that affects $\mathrm{CA}$ is the neurogenic mechanisms which is associated with the nervous system, including the performance of the autonomic nervous system. The autonomic nervous system works involuntarily to control breathing, digestion, heart rate, blood pressure, and others. The nervous system is divided into the sympathetic and the parasympathetic nervous system.

The activity between the sympathetic nervous system and parasympathetic can be seen through heart rate variability. Heart rate variability (HRV) can quickly indicate disruption in the autonomic nervous system [12]. Disruption of the balance in the autonomic nervous system certainly affects the mechanism in CA. It is therefore important to look at HRV as a parameter to determine the balance of the 
autonomic nervous system associated with CA mechanisms in stroke ischemic sufferers.

This research has hypothesis that the disruption of the autonomic nervous system in patients with ischemic stroke can be seen through the parameters contained in HRV. Blood pressure and blood flow velocity also affects the mechanism of CA both in stroke patients and non-stroke. Therefore, an understanding of the physiological conditions of elderly ischemic stroke patients in terms of blood pressure, blood flow velocity, and heart rate variability is needed.

\section{MATERIALS AND METHODS}

\subsection{Subjects}

The dataset used in this study is a secondary dataset [13] obtained from the Syncope and Falls in the Elderly Laboratory and the Magnetic Resonance Imaging Center at Beth Israel Deaconess Medical Center (BIDMC) [14]. This dataset consists of 40 patients divided into two groups, 20 patients with stroke and 20 non-strokes (control). The subjects of this study are 45-80 years old.

The stroke group consisted of patients suffering from ischemic stroke for 6 months and in a stable clinical condition, confirmed by neurological examination with a modified Rankin Scale $(\mathrm{mRS})<4$, and the National Institute Health Stroke Score (NIHSS) $<5$ at the time of the study. The scale of MRS $<4$ and NIHSS $<5$ indicate that the patient can still respond to two tasks correctly despite a decrease in sensory, motoric, and can walk without assistance. Patients without a clinical history of stroke (control) do not experience a decrease in focus during neurological examination.

Patients who were not included in the study were patients with subarachnoid hemorrhage [15], diabetes, arrhythmias [16], atrial fibrillation, dementia, obesity with a Body Mass Index $(\mathrm{BMI})>35$ [17], acute medical conditions, alcohol and drug abuse, and severe hypertension (systole > 200 diastole $>110$ or patients with 3 or more anti-hypertension) [18].

\subsection{Experimental Design}

Subjects were taken by sitting, standing, supine (baseline), and tilted positions. BP is recorded using Dynamap placed on the upper arm and BFV is recorded using Transcranial Doppler (TCD) placed on the right and left Middle Cerebral Artery (MCA).

ECG data is retrieved using the ME6000 (MegaElectronics) 12 leads device. Records during the baseline and tilt positions are taken for 10 minutes. Sit-stand position is taken with the protocol for 5 minutes sitting with eyes open (SitEO), 5 minutes standing in the open eyes (StandEO), then 5 minutes sitting with eyes closed (SitEC) and 5 minutes standing in a closed eyes (StandEC). Each position is taken with a $1000 \mathrm{~Hz}$ sampling rate.

\subsection{Signal Processing and Features Extraction}

ECG data is processed using Python 3.7 signal processing tools to get the R Peak in the stroke and control groups. The R-peak on the ECG signal in each subject is detected using the Two-Moving Average Method. Detection results for each R-Peak are accommodated simultaneously with the position of the $\mathrm{x}$-axis. The detected R-Peak position is then used to calculate the distance between the R-Peak (R$\mathrm{R}$ interval).

The R-R interval is obtained by calculating the difference in the position of the R-Peak with the R-Peak position afterwards. After getting the R-R interval, new time series data is obtained. This time series data is then used for HRV parameter analysis.

HRV parameters in the time domain include: MeanRR, SDRR, RMSSD, and CVRR. In the Poincare Plot, the parameters used are SD1 and SD2. Analysis of the frequency domain begins with transforming time series data into the form of frequency functions using the pyhrv package in python. This step starts by checking the value of the R-R interval, then interpolating with a sampling frequency of 4 $\mathrm{Hz}$. The interpolation signal is then computed by Power Spectral Density (PSD) and its parameters (VLF, LF, HF, and $\mathrm{LF} / \mathrm{HF}$ ratio).

\subsection{Data Analysis}

The data in this study are not normally distributed, so the average test (significance test) is using non-parametric tests. The Mann-Whitney U Method is a non-parametric test that can provide the most accurate averaging estimate, when the sample size is small, and the data are not normally distributed [19]. This method can be used to provide significant values on Waist-Hip Ratio (WHR), Body Mass Index (BMI), and HRV data between the obese and normal groups with a sample size $<30$ in each group [20]. Because the data of this study are not normally distributed and the sample data is $<30$ in each group, the significance test in this study uses the Mann-Whitney U Method.

Spearman Rank Correlation Test was conducted to determine the relationship between BP parameters, BFV, and HRV in each group. Data are presented using mean \pm standard deviations (Mean $\pm \mathrm{SD}$ ). The significant value ( $\mathrm{p}$ value) used in this study is 0.05 . The magnitude of the correlation is expressed in the coefficient correlation (r). All statistical tests were performed using SPSS Software version 15.0.

\section{Results}

\subsection{Demography}

Descriptive statistics are used to summarize all variables in the subject demographics. Table 1 shows a demographic summary of each groups. There was no difference in age and systolic blood pressure between the stroke and control groups ( $\mathrm{p}$-value >0.05). Likewise, with the right and left 
TABLE 1

SUBJECT DEMOGRAPHY

\begin{tabular}{lccc}
\hline \hline \multicolumn{1}{c}{ Demography } & $\begin{array}{c}\text { Stroke } \\
(\text { Mean } \pm \text { SD) }\end{array}$ & $\begin{array}{c}\text { Control } \\
(\text { Mean } \pm \text { SD) }\end{array}$ & p-value \\
\hline Age & $64 \pm 10.11$ & $67 \pm 7.53$ & 0.273 \\
Gender (M/F) & $7 / 13$ & $9 / 11$ & 0.524 \\
Body Mass Index (kg/m) & $28.34 \pm 3.83$ & $25.15 \pm 4.57$ & $\mathbf{0 . 0 1 2} *$ \\
SBP (mmHg) & $135.62 \pm 8.58$ & $130.43 \pm 11.85$ & 0.168 \\
DBP (mmHg) & $69.42 \pm 5.84$ & $63.48 \pm 5.75$ & $\mathbf{0 . 0 0 3 *}$ \\
Right MCA Diameter & $2.13 \pm 0.64$ & $2.38 \pm 0.23$ & 0.240 \\
Left MCA Diameter & $2.48 \pm 0.28$ & $2.43 \pm 0.22$ & 0.762 \\
Smoking History (Y/N) & $13 / 7$ & $7 / 13$ & 0.061 \\
Alcohol History (Y/N) & $15 / 5$ & $16 / 4$ & 0.708 \\
NIHSS & $3 \pm 2.28$ & - & - \\
mRS & $1 \pm 1.08$ & - & - \\
Stroke Side (Right/Left) & $8 / 12$ & - & - \\
\hline \hline
\end{tabular}

* Significant level 0.05

$S D=$ Standard Deviation, $M=$ Male, $F=$ Female, $S B P=$ Systolic Blood Pressure, $D B P=$ Diastolic Blood Pressure, $M C A=$ Middle Cerebral Artery, $Y=Y e s, N=N o$ NIHSS=National Institute Health Stroke Score, $m R S=$ modified Rankin Score.

MCA diameters, smoking history, and alcohol history. In demographics of body mass index and diastolic blood pressure, the mean in the stroke group tended to be higher than in the control group. Regarding differences in body mass index and diastolic blood pressure in the two groups will be discussed in the discussion section

\subsection{Blood Pressure}

Blood Pressure's significance test for the stroke and control groups are shown in Table 2. Based on these results, BP in each position has a p-value $>0.05$. This means that there is no significant difference in BP average between the two groups. Mean BP was shown as Mean Arterial Pressure (MAP).

Although there is no significant difference between the stroke and control groups, the BP average in the stroke group is always higher than the control group. The BP average in the stroke group increased by 3-7 $\mathrm{mmHg}$ (Fig. 1). In addition, the average BP in both groups also increased when there was a change in position from the baseline.

\subsection{Blood Flow Velocity}

Blood Flow Velocity results between stroke group and the control group showed no significant difference. Table 3 shows the results of the BFV significance test, where there

TABLE 3

BLOOD Flow Velocity SigNIFICANCE TEST

\begin{tabular}{lccc}
\hline \hline \multicolumn{1}{c}{ Position } & $\begin{array}{c}\text { Control } \\
\text { (Mean } \pm \text { SD) }\end{array}$ & $\begin{array}{c}\text { Stroke } \\
(\text { Mean } \pm \text { SD) }\end{array}$ & p-value \\
\hline Baseline & $46.37 \pm 16.15$ & $40.99 \pm 23.94$ & 0.226 \\
Tilt & $42.97 \pm 14.17$ & $40.22 \pm 22.24$ & 0.389 \\
Sit Eyes Open & $41.54 \pm 12.48$ & $37.98 \pm 16.71$ & 0.352 \\
Stand Eyes Open & $40.29 \pm 14.04$ & $36.95 \pm 17.08$ & 0.270 \\
Sit Eyes Close & $39.51 \pm 12.27$ & $35.20 \pm 16.51$ & 0.168 \\
Stand Eyes Close & $36.81 \pm 12.15$ & $34.27 \pm 17.70$ & 0.368 \\
\hline \hline
\end{tabular}

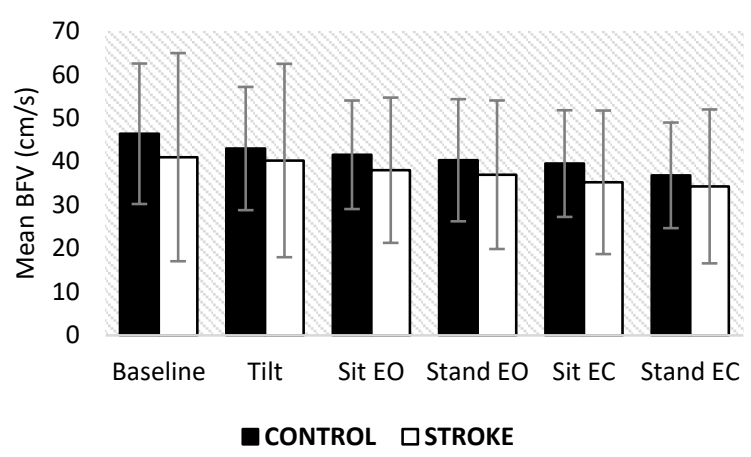

Fig. 2. Mean Blood Flow Velocity Comparison

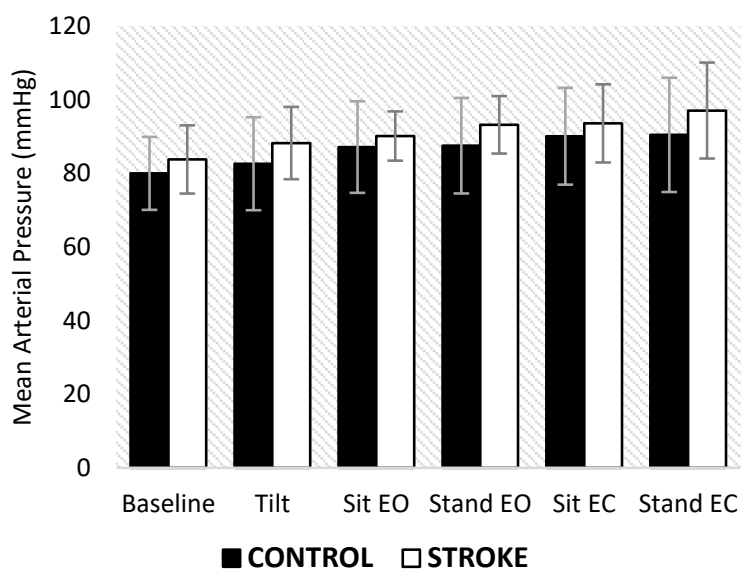

Fig. 1. Mean Arterial Pressure Comparison

TABLE 2

BloOd PRESSURE SigNIFICANCE TEST

\begin{tabular}{lccc}
\hline \hline \multicolumn{1}{c}{ Position } & $\begin{array}{c}\text { Control } \\
\text { (Mean } \pm \text { SD) }\end{array}$ & $\begin{array}{c}\text { Stroke } \\
(\text { Mean } \pm \text { SD) }\end{array}$ & p-value \\
\hline Baseline & $79.99 \pm 9.90$ & $83.78 \pm 9.27$ & 0.219 \\
Tilt & $82.61 \pm 12.63$ & $88.23 \pm 9.82$ & 0.128 \\
Sit Eyes Open & $87.14 \pm 12.44$ & $90.14 \pm 6.69$ & 0.372 \\
Stand Eyes Open & $87.52 \pm 12.98$ & $93.18 \pm 7.80$ & 0.120 \\
Sit Eyes Close & $90.08 \pm 13.15$ & $93.57 \pm 10.62$ & 0.392 \\
Stand Eyes Close & $90.45 \pm 15.52$ & $97.06 \pm 13.03$ & 0.186 \\
\hline \hline
\end{tabular}

was no significant difference between the stroke and control groups.

The mean BFV in the stroke group was lower than the control group (Fig. 2) in any positions. If the average BP has increased when there is a change in position, this applies in reverse to the BFV.

\subsection{Heart Rate Variability}

HRV analysis is performed in time domain, frequency domain, and Poincaré Plot. Analysis in each domain is useful 
TABLE 5

HEART RATE VARIABILITY (TIME DOMAIN) SigNIFICANCE TEST

\begin{tabular}{lccc}
\hline \hline \multicolumn{1}{c}{ Position } & $\begin{array}{c}\text { Control } \\
\text { (Mean } \pm \text { SD) }\end{array}$ & $\begin{array}{c}\text { Stroke } \\
\text { (Mean } \pm \text { SD) }\end{array}$ & p-value \\
\hline MeanRR Base & $484.69 \pm 67.56$ & $429.83 \pm 39.61$ & $\mathbf{0 . 0 0 3 *}$ \\
MeanRR Tilt & $428.63 \pm 70.57$ & $378.00 \pm 38.59$ & $\mathbf{0 . 0 2 5} *$ \\
MeanRR SitEO & $944.11 \pm 160.67$ & $842.95 \pm 69.15$ & $\mathbf{0 . 0 2 7} *$ \\
MeanRR StandEO & $844.85 \pm 152.74$ & $744.28 \pm 84.10$ & $\mathbf{0 . 0 4 2} *$ \\
MeanRR SitEC & $954.42 \pm 165.74$ & $832.74 \pm 75.76$ & $\mathbf{0 . 0 0 7} *$ \\
MeanRR StandEC & $831.46 \pm 164.47$ & $731.48 \pm 88.70$ & 0.079 \\
\hline SDRR Base & $36.34 \pm 11.68$ & $23.32 \pm 8.83$ & $<\mathbf{0 . 0 0 1} * *$ \\
SDRR Tilt & $18.26 \pm 7.00$ & $15.62 \pm 6.69$ & 0.279 \\
SDRR SitEO & $36.30 \pm 18.70$ & $29.45 \pm 10.21$ & 0.204 \\
SDRR StandEO & $32.15 \pm 13.39$ & $23.80 \pm 13.30$ & $\mathbf{0 . 0 0 9 *}$ \\
SDRR SitEC & $37.10 \pm 15.45$ & $34.10 \pm 15.04$ & 0.433 \\
SDRR StandEC & $32.66 \pm 20.35$ & $25.49 \pm 11.54$ & 0.267 \\
\hline RMSSD Base & $24.86 \pm 16.12$ & $16.06 \pm 10.92$ & 0.094 \\
RMSSD Tilt & $13.46 \pm 11.54$ & $12.02 \pm 10.84$ & 0.745 \\
RMSSD SitEO & $30.44 \pm 27.55$ & $27.34 \pm 20.74$ & 0.850 \\
RMSSD StandEO & $25.18 \pm 25.04$ & $19.34 \pm 20.54$ & 0.787 \\
RMSSD SitEC & $33.95 \pm 27.91$ & $27.51 \pm 20.55$ & 0.344 \\
RMSSD StandEC & $30.56 \pm 36.91$ & $23.76 \pm 22.46$ & 0.914 \\
\hline CVRR Base & $0.074 \pm 0.020$ & $0.054 \pm 0.019$ & $\mathbf{0 . 0 0 2 *}$ \\
CVRR Tilt & $0.043 \pm 0.016$ & $0.041 \pm 0.018$ & 0.589 \\
CVRR SitEO & $0.038 \pm 0.017$ & $0.035 \pm 0.013$ & 0.797 \\
CVRR StandEO & $0.038 \pm 0.017$ & $0.032 \pm 0.018$ & 0.076 \\
CVRR SitEC & $0.039 \pm 0.017$ & $0.041 \pm 0.018$ & 0.766 \\
CVRR StandEC & $0.040 \pm 0.025$ & $0.035 \pm 0.016$ & 0.636 \\
\hline \hline
\end{tabular}

to find out the differences in HRV in the stroke and control groups.

HRV parameters used in the time domain are MeanRR, SDRR, RMSSD, and CVRR. Significance test shows that each HRV parameter in the two groups gave different significant results (Table 4). Almost all p-values in the MeanRR parameter are smaller than 0.05 for each position, except for the StandEC position ( $p$-value> 0.5). This means that commonly there are significant differences between the stroke and control groups in the MeanRR parameters.

In the SDRR parameters, significant differences in stroke and control groups were only seen at the baseline and StandEO positions ( $\mathrm{p}$-value <0.05). Apart from these two positions, the SDRR parameter did not show a significant difference ( $p$-value> 0.5) among the two groups.

The RMSSD parameters in the significance test did not show a significant difference between the stroke and control groups in all positions ( $\mathrm{p}$-value> 0.05). This means that the mean RMSSD in the two groups is the same. The CVRR parameter only shows a significant difference in the baseline position ( $\mathrm{p}$-value <0.05).

Significance tests were carried out on both groups, to determine the difference in the frequency domain in each parameter/powerband. The results of the significance tests are shown in Table 5. In the VLF parameter a significant difference is shown in the baseline position and stand eyes open ( $\mathrm{p}$-value $<0.05$ ). Other parameters that showed significant differences between the two groups were the LF parameter at the baseline position and sit eyes open, and the
TABLE 4

HEART RATE VARIABILITy (FREQUENCY DOMAIN) SIGNIFICANCE TEST

\begin{tabular}{lccc}
\hline \hline \multicolumn{1}{c}{ Position } & $\begin{array}{c}\text { Control } \\
\text { (Mean } \pm \text { SD) }\end{array}$ & $\begin{array}{c}\text { Stroke } \\
\text { (Mean } \pm \text { SD) }\end{array}$ & p-value \\
\hline VLF Base & $344.93 \pm 188.97$ & $165.68 \pm 140.76$ & $\mathbf{0 . 0 0 2 *}$ \\
VLF Tilt & $134.16 \pm 151.54$ & $102.47 \pm 120.84$ & 0.607 \\
VLF SitEO & $505.11 \pm 680.32$ & $234.93 \pm 130.21$ & 0.117 \\
VLF StandEO & $503.16 \pm 346.10$ & $268.88 \pm 294.29$ & $\mathbf{0 . 0 0 9 *}$ \\
VLF SitEC & $507.23 \pm 576.16$ & $454.32 \pm 328.32$ & 0.978 \\
VLF StandEC & $262.50 \pm 196.69$ & $198.40 \pm 118.31$ & 0.516 \\
\hline LF Base & $206.64 \pm 87.14$ & $103.37 \pm 74.77$ & $<\mathbf{0 . 0 0 1 * *}$ \\
LF Tilt & $112.20 \pm 214.38$ & $82.89 \pm 179.50$ & 0.745 \\
LF SitEO & $422.04 \pm 691.69$ & $133.36 \pm 103.89$ & $\mathbf{0 . 0 0 6 *}$ \\
LF StandEO & $362.21 \pm 638.60$ & $211.01 \pm 476.07$ & 0.144 \\
LF SitEC & $321.55 \pm 349.06$ & $314.35 \pm 480.79$ & 0.344 \\
LF StandEC & $226.28 \pm 320.78$ & $129.92 \pm 127.05$ & 0.766 \\
\hline HF Base & $152.69 \pm 146.62$ & $59.41 \pm 52.96$ & $\mathbf{0 . 0 0 9 *}$ \\
HF Tilt & $48.02 \pm 85.42$ & $78.93 \pm 255.15$ & 0.160 \\
HF SitEO & $391.79 \pm 661.78$ & $234.32 \pm 290.56$ & 0.935 \\
HF StandEO & $395.14 \pm 919.35$ & $238.39 \pm 691.09$ & 0.808 \\
HF SitEC & $441.30 \pm 691.82$ & $378.90 \pm 659.30$ & 0.372 \\
HF StandEC & $561.27 \pm 1812.36$ & $149.35 \pm 221.66$ & 0.871 \\
\hline LF/HF Base & $2.292 \pm 2.073$ & $2.216 \pm 1.260$ & 0.552 \\
LF/HF Tilt & $2.589 \pm 3.355$ & $2.773 \pm 2.659$ & 0.646 \\
LF/HF SitEO & $2.887 \pm 3.211$ & $1.076 \pm 0.788$ & 0.105 \\
LF/HF StandEO & $4.262 \pm 5.982$ & $1.725 \pm 1.211$ & 0.402 \\
LF/HF SitEC & $2.241 \pm 2.942$ & $1.622 \pm 1.551$ & 0.978 \\
LF/HF StandEC & $2.902 \pm 4.400$ & $1.719 \pm 2.333$ & 0.935 \\
\hline \hline
\end{tabular}

HF parameter at the baseline position. The LF/HF Ratio did not show any significant difference between the two groups.

Generally, through the significance test, more significant differences in various positions are shown in the VLF and LF parameters. However, the stroke group with the control group at the baseline position showed the most significant difference in all power bands.

To find out the differences in HRV through non-linear analysis (Poincaré Plot) between the stroke group and the control group, a significance test was performed on the parameters SD1 and SD2. Poincaré Plot significance test results for each parameter in various positions as shown in Table 6.

TABLE 6

Heart Rate VARIABILITy (POINCARÉ PLOT) SignificAnCE TEST

\begin{tabular}{lccc}
\hline \hline \multicolumn{1}{c}{ Position } & $\begin{array}{c}\text { Control } \\
(\text { Mean } \pm \text { SD) }\end{array}$ & $\begin{array}{c}\text { Stroke } \\
(\text { Mean } \pm \text { SD) }\end{array}$ & p-value \\
\hline SD1 Base & $17.58 \pm 11.40$ & $11.36 \pm 7.72$ & 0.094 \\
SD1 Tilt & $9.52 \pm 8.17$ & $8.50 \pm 7.67$ & 0.745 \\
SD1 SitEO & $21.56 \pm 19.52$ & $19.36 \pm 14.69$ & 0.850 \\
SD1 StandEO & $17.85 \pm 17.75$ & $13.70 \pm 14.55$ & 0.787 \\
SD1 SitEC & $24.05 \pm 19.77$ & $19.48 \pm 14.55$ & 0.344 \\
SD1 StandEC & $21.67 \pm 26.17$ & $16.84 \pm 15.92$ & 0.914 \\
\hline SD2 Base & $47.60 \pm 14.57$ & $30.29 \pm 11.81$ & $<\mathbf{0 . 0 0 1 * *}$ \\
SD2 Tilt & $23.26 \pm 8.25$ & $19.88 \pm 7.19$ & 0.245 \\
SD2 SitEO & $45.35 \pm 20.94$ & $35.61 \pm 9.45$ & 0.088 \\
SD2 StandEO & $40.33 \pm 13.09$ & $30.01 \pm 13.73$ & $\mathbf{0 . 0 0 6 *}$ \\
SD2 SitEC & $44.96 \pm 15.76$ & $43.08 \pm 18.33$ & 0.516 \\
SD2 StandEC & $38.57 \pm 18.12$ & $30.67 \pm 9.59$ & 0.279 \\
\hline \hline
\end{tabular}


TABLE 7

BP VS BFV CORRELATION

\begin{tabular}{lccccc}
\hline \multirow{2}{*}{ Position } & \multicolumn{2}{c}{ Control Group } & \multicolumn{2}{c}{ Stroke Group } \\
\cline { 2 - 6 } & & SBP & DBP & SBP & DBP \\
\hline \multirow{2}{*}{ Baseline } & $\mathrm{r}$ & -0.388 & -0.100 & -0.039 & 0.254 \\
& $\mathrm{p}$-value & 0.101 & 0.684 & 0.875 & 0.293 \\
\hline \multirow{2}{*}{ Tilt } & $\mathrm{r}$ & 0.049 & 0.019 & -0.274 & 0.047 \\
& $\mathrm{p}$-value & 0.842 & 0.937 & 0.257 & 0.847 \\
\hline \multirow{2}{*}{ SitEO } & $\mathrm{r}$ & -0.485 & -0.288 & 0.296 & -0.042 \\
& $\mathrm{p}$-value & 0.057 & 0.279 & 0.233 & 0.868 \\
\multirow{2}{*}{ StandEO } & $\mathrm{r}$ & -0.218 & -0.147 & 0.323 & -0.071 \\
& $\mathrm{p}$-value & 0.418 & 0.587 & 0.191 & 0.779 \\
\multirow{2}{*}{ SitEC } & $\mathrm{r}$ & $\mathbf{- 0 . 5 7 4}$ & -0.465 & 0.176 & -0.03 \\
& $\mathrm{p}$-value & $\mathbf{0 . 0 2 0}$ & 0.070 & 0.484 & 0.906 \\
\hline \multirow{2}{*}{ StandEC } & $\mathrm{r}$ & -0.212 & -0.247 & 0.301 & 0.238 \\
& $\mathrm{p}$-value & 0.431 & 0.356 & 0.240 & 0.358 \\
\hline \hline
\end{tabular}

* p-value $<0.05$, SBP=Systolic Blood Pressure, DBP=Diastolic Blood

Pressure, $r=$ Coefficient Correlation, $p$-value=Significance Level

There was no significant difference in the SD1 parameter ( $p$-value $>0.05$ ) in all positions. Different results are shown in SD2 parameters which indicate that there are significant differences between the two groups. This condition is found only in the baseline and StandEO positions

\subsection{Blood Pressure and Blood Flow Velocity}

The Spearman Correlation Rank Test was used to determine the relationship between $\mathrm{BP}$ and $\mathrm{BFV}$ in both groups. The correlation test results in Table 7 show that in the control group, a relationship was found between $\mathrm{BP}$ and BFV at the SitEC position ( $\mathrm{p}$-value $<0.05)$. The correlation shown is a negative correlation with a correlation coefficient of 0.574 , which means that there is a strong correlation between BP and BFV in the control group. Different results are shown in the stroke group. In the stroke group, there was no relationship between $\mathrm{BP}$ and $\mathrm{BFV}$ in various positions with a p-value $>0.05$.

\subsection{Blood Pressure and Heart Rate Variability}

The association between BP and HRV was also tested using the same method as the BP test with BFV. Correlation test results between $\mathrm{BP}$ and time domain $\mathrm{HRV}$ in the control group are shown in Table 8 . As seen in the table, the results of the correlation test between BP and HRV time domains in all positions in the control group have p-value $>0.05$. This means that the control group did not show any relationship between BP and HRV in the time domain. Each HRV parameter in the frequency domain (VLF, LF, HF, and LF/HF Ratio) did not have any correlation with BP in the control group (p-value> 0.05). HRV on the Poincaré Plot is shown with parameters SD1 and SD2. In the control group, no relationship was found between BP and the two parameters in all positions. The significance value indicated more than 0.05 (p-value > 0.05).

Correlation test in the stroke group gives different results in several positions (Table 9). Baseline, tilt, sit eyes open, and stand eyes open positions do not indicate a relationship. However, in sit eyes close and stand eyes close position, the parameters SDRR, RMSSD, and CVRR have a correlation with BP (p-value <0.05). The direction of the positive correlation is indicated between these parameters with BP, with a strong correlation level $(r>0.5)$. Correlation test between BP and HRV frequency domain showed an association (Table 9). The correlation coefficient (r) on the

TABLE 8

BP VS HRV CORRELATION CONTROL GROUP

\begin{tabular}{|c|c|c|c|c|c|c|c|c|c|c|c|c|c|}
\hline \multirow{2}{*}{\multicolumn{2}{|c|}{ Blood Pressure }} & \multicolumn{2}{|c|}{ Baseline } & \multicolumn{2}{|c|}{ Tilt } & \multicolumn{2}{|c|}{ SitEO } & \multicolumn{2}{|c|}{ StandEO } & \multicolumn{2}{|c|}{ SitEC } & \multicolumn{2}{|c|}{ StandEC } \\
\hline & & SDP & DBP & SDP & DBP & SDP & DBP & SDP & DBP & SDP & DBP & SDP & DBP \\
\hline \multirow{2}{*}{ MeanRR } & $\mathrm{r}$ & -0.021 & -0.108 & 0.381 & 0.042 & 0.168 & -0.2 & 0.326 & -0.218 & 0.271 & -0.291 & 0.282 & -0.247 \\
\hline & p-value & 0.93 & 0.65 & 0.108 & 0.864 & 0.535 & 0.458 & 0.217 & 0.418 & 0.311 & 0.274 & 0.289 & 0.356 \\
\hline \multirow{2}{*}{ SDRR } & $\mathrm{r}$ & 0.238 & -0.023 & -0.032 & 0.033 & -0.115 & -0.209 & 0.097 & -0.026 & 0.218 & -0.018 & -0.147 & -0.212 \\
\hline & $\mathrm{p}$-value & 0.313 & 0.925 & 0.898 & 0.892 & 0.672 & 0.438 & 0.721 & 0.922 & 0.418 & 0.948 & 0.587 & 0.431 \\
\hline \multirow{2}{*}{ RMSSD } & $\mathrm{r}$ & 0.311 & -0.017 & 0.298 & 0.14 & 0.238 & -0.171 & 0.126 & -0.194 & 0.324 & -0.185 & 0.147 & -0.094 \\
\hline & p-value & 0.182 & 0.945 & 0.215 & 0.567 & 0.374 & 0.528 & 0.641 & 0.471 & 0.222 & 0.492 & 0.587 & 0.729 \\
\hline \multirow{2}{*}{ CVRR } & $\mathrm{r}$ & 0.324 & 0.06 & -0.135 & 0.093 & -0.2 & -0.229 & 0.038 & 0.085 & 0.135 & 0.112 & -0.291 & -0.197 \\
\hline & p-value & 0.163 & 0.801 & 0.581 & 0.705 & 0.458 & 0.393 & 0.888 & 0.753 & 0.617 & 0.68 & 0.274 & 0.464 \\
\hline \multirow{2}{*}{ VLF } & $\mathrm{r}$ & -0.006 & -0.269 & -0.175 & -0.047 & -0.244 & -0.268 & 0.224 & -0.118 & 0.335 & 0.074 & -0.029 & -0.003 \\
\hline & $\mathrm{p}$-value & 0.98 & 0.251 & 0.473 & 0.847 & 0.362 & 0.316 & 0.405 & 0.664 & 0.204 & 0.787 & 0.914 & 0.991 \\
\hline \multirow{2}{*}{$\mathrm{LF}$} & $\mathrm{r}$ & 0.146 & -0.074 & -0.421 & -0.237 & -0.115 & -0.062 & 0.182 & 0.05 & 0.071 & 0.032 & 0.156 & 0.021 \\
\hline & p-value & 0.539 & 0.758 & 0.073 & 0.329 & 0.672 & 0.82 & 0.499 & 0.854 & 0.795 & 0.905 & 0.564 & 0.94 \\
\hline \multirow{2}{*}{$\mathrm{HF}$} & $\mathrm{r}$ & 0.325 & -0.027 & -0.295 & 0.021 & 0.185 & -0.268 & 0.168 & -0.235 & 0.312 & -0.262 & 0.332 & -0.044 \\
\hline & p-value & 0.162 & 0.91 & 0.221 & 0.932 & 0.492 & 0.316 & 0.535 & 0.38 & 0.24 & 0.327 & 0.208 & 0.871 \\
\hline \multirow{2}{*}{ LF/HF Ratio } & $\mathrm{r}$ & -0.26 & -0.12 & -0.151 & -0.298 & -0.362 & 0.162 & -0.25 & 0.226 & -0.197 & 0.406 & -0.422 & 0.128 \\
\hline & p-value & 0.268 & 0.613 & 0.538 & 0.215 & 0.169 & 0.549 & 0.35 & 0.399 & 0.464 & 0.119 & 0.103 & 0.637 \\
\hline \multirow{2}{*}{ SD1 } & $\mathrm{r}$ & 0.311 & -0.017 & 0.298 & 0.14 & 0.238 & -0.171 & 0.126 & -0.194 & 0.324 & -0.185 & 0.147 & -0.094 \\
\hline & p-value & 0.182 & 0.945 & 0.215 & 0.567 & 0.374 & 0.528 & 0.641 & 0.471 & 0.222 & 0.492 & 0.587 & 0.729 \\
\hline \multirow{2}{*}{$\mathrm{SD} 2$} & $\mathrm{r}$ & 0.167 & -0.045 & -0.132 & 0.005 & -0.247 & -0.268 & 0.047 & -0.029 & 0.065 & -0.035 & -0.079 & -0.115 \\
\hline & p-value & 0.482 & 0.85 & 0.591 & 0.983 & 0.356 & 0.316 & 0.863 & 0.914 & 0.812 & 0.897 & 0.77 & 0.672 \\
\hline
\end{tabular}

* p-value $<0.05$, SBP=Systolic Blood Pressure, DBP=Diastolic Blood Pressure, $r=$ Coefficient Correlation, $p$-value $=$ Significance Level 
TABLE 10

BP VS HRV CORRELATION STROKE GROUP

\begin{tabular}{|c|c|c|c|c|c|c|c|c|c|c|c|c|c|}
\hline \multirow{2}{*}{\multicolumn{2}{|c|}{ Blood Pressure }} & \multicolumn{2}{|c|}{ Baseline } & \multicolumn{2}{|c|}{ Tilt } & \multicolumn{2}{|c|}{ SitEO } & \multicolumn{2}{|c|}{ StandEO } & \multicolumn{2}{|c|}{ SitEC } & \multicolumn{2}{|c|}{ StandEC } \\
\hline & & SDP & DBP & SDP & DBP & SDP & DBP & SDP & DBP & SDP & DBP & SDP & DBP \\
\hline \multirow{2}{*}{ MeanRR } & $\mathrm{r}$ & -0.259 & -0.093 & 0.256 & 0 & -0.154 & 0.096 & -0.065 & 0.065 & 0.214 & 0.081 & 0.381 & 0.201 \\
\hline & p-value & 0.271 & 0.696 & 0.277 & 1 & 0.528 & 0.694 & 0.792 & 0.792 & 0.379 & 0.743 & 0.119 & 0.423 \\
\hline \multirow{2}{*}{ SDRR } & $r$ & -0.242 & -0.024 & 0.065 & -0.168 & 0.281 & 0.293 & 0.081 & 0.005 & 0.481 & 0.16 & 0.616 & 0.143 \\
\hline & p-value & 0.304 & 0.92 & 0.787 & 0.478 & 0.244 & 0.223 & 0.743 & 0.983 & $0.037 *$ & 0.514 & 0.006* & 0.57 \\
\hline \multirow{2}{*}{ RMSSD } & $\mathrm{r}$ & 0.122 & -0.164 & 0.198 & -0.229 & 0.219 & 0.074 & 0.016 & 0.161 & 0.4 & -0.03 & 0.542 & 0.088 \\
\hline & $\mathrm{p}$-value & 0.609 & 0.49 & 0.402 & 0.332 & 0.367 & 0.764 & 0.949 & 0.509 & 0.09 & 0.904 & $0.020 *$ & 0.729 \\
\hline \multirow{2}{*}{ CVRR } & $\mathrm{r}$ & -0.173 & 0.036 & 0.026 & -0.119 & 0.268 & 0.344 & 0.126 & 0.144 & 0.461 & 0.158 & 0.531 & 0.132 \\
\hline & p-value & 0.466 & 0.88 & 0.915 & 0.618 & 0.267 & 0.149 & 0.606 & 0.557 & 0.047* & 0.519 & 0.023* & 0.601 \\
\hline \multirow{2}{*}{ VLF } & $\mathrm{r}$ & -0.232 & -0.074 & -0.032 & -0.179 & 0.2 & 0.419 & 0.096 & -0.044 & 0.305 & 0.393 & 0.259 & -0.146 \\
\hline & p-value & 0.326 & 0.758 & 0.895 & 0.45 & 0.412 & 0.074 & 0.694 & 0.858 & 0.204 & 0.096 & 0.299 & 0.565 \\
\hline \multirow{2}{*}{ LF } & $\mathrm{r}$ & -0.275 & 0.008 & -0.179 & -0.338 & 0.246 & 0.56 & 0.005 & -0.346 & 0.274 & 0.111 & 0.214 & 0.055 \\
\hline & $\mathrm{p}$-value & 0.24 & 0.975 & 0.45 & 0.145 & 0.311 & 0.013* & 0.983 & 0.147 & 0.257 & 0.652 & 0.395 & 0.829 \\
\hline \multirow{2}{*}{$\mathrm{HF}$} & $\mathrm{r}$ & -0.269 & -0.104 & -0.438 & -0.385 & 0.2 & 0.139 & -0.079 & -0.232 & 0.309 & -0.077 & 0.391 & 0.104 \\
\hline & p-value & 0.251 & 0.663 & 0.054 & 0.094 & 0.412 & 0.571 & 0.748 & 0.34 & 0.198 & 0.753 & 0.108 & 0.681 \\
\hline \multirow{2}{*}{ LF/HF Ratio } & $\mathrm{r}$ & -0.002 & 0.041 & 0.427 & 0.183 & -0.133 & 0.1 & 0.107 & 0.002 & -0.074 & 0.142 & -0.259 & 0.02 \\
\hline & p-value & 0.995 & 0.865 & 0.06 & 0.439 & 0.586 & 0.684 & 0.663 & 0.994 & 0.764 & 0.562 & 0.299 & 0.938 \\
\hline \multirow{2}{*}{ SD1 } & $\mathrm{r}$ & 0.122 & -0.164 & 0.198 & -0.229 & 0.219 & 0.074 & 0.016 & 0.161 & 0.4 & -0.03 & 0.542 & 0.088 \\
\hline & p-value & 0.609 & 0.49 & 0.402 & 0.332 & 0.367 & 0.764 & 0.949 & 0.509 & 0.09 & 0.904 & 0.020* & 0.729 \\
\hline \multirow{2}{*}{ SD2 } & $\mathrm{r}$ & -0.352 & -0.009 & -0.086 & -0.232 & 0.344 & 0.346 & 0.044 & 0.025 & 0.377 & 0.109 & 0.467 & 0.102 \\
\hline & p-value & 0.128 & 0.97 & 0.719 & 0.326 & 0.149 & 0.147 & 0.858 & 0.92 & 0.111 & 0.658 & 0.05 & 0.687 \\
\hline
\end{tabular}

* $p$-value $<0.05, S B P=$ Systolic Blood Pressure, DBP=Diastolic Blood Pressure, $r=$ Coefficient Correlation, $p$-value=Significance Level

TABLE 9

BFV VS HRV CORRELATION

\begin{tabular}{|c|c|c|c|c|c|c|c|c|c|c|c|c|c|}
\hline \multirow{2}{*}{\multicolumn{2}{|c|}{$\begin{array}{l}\text { Blood Flow } \\
\text { Velocity }\end{array}$}} & \multicolumn{6}{|c|}{ Control Group } & \multicolumn{6}{|c|}{ Stroke Group } \\
\hline & & \multirow{2}{*}{$\begin{array}{c}\text { Baseline } \\
0.146 \\
\end{array}$} & \multirow{2}{*}{$\frac{\text { Tilt }}{0.344}$} & \multirow{2}{*}{$\begin{array}{c}\text { SitEO } \\
-0.012\end{array}$} & \multirow{2}{*}{$\begin{array}{c}\begin{array}{c}\text { Stand } \\
\text { EO }\end{array} \\
0.029\end{array}$} & \multirow{2}{*}{$\begin{array}{c}\text { SitEC } \\
-0.024\end{array}$} & \multirow{2}{*}{$\begin{array}{c}\text { Stand } \\
\text { EC }\end{array}$} & \multirow{2}{*}{$\begin{array}{c}\text { Baseline } \\
-0.107\end{array}$} & \multirow{2}{*}{$\frac{\text { Tilt }}{-0.033}$} & \multirow{2}{*}{$\begin{array}{c}\text { SitEO } \\
0.135\end{array}$} & \multirow{2}{*}{$\begin{array}{c}\begin{array}{c}\text { Stand } \\
\text { EO }\end{array} \\
0.119\end{array}$} & \multirow{2}{*}{$\begin{array}{r}\text { SitEC } \\
0.181\end{array}$} & \multirow{2}{*}{$\begin{array}{c}\begin{array}{c}\text { Stand } \\
\text { EC }\end{array} \\
0.284\end{array}$} \\
\hline MeanPP & $\mathrm{r}$ & & & & & & & & & & & & \\
\hline MeanKR & $\mathrm{p}$-value & 0.552 & 0.149 & 0.966 & 0.914 & 0.931 & 0.974 & 0.663 & 0.892 & 0.593 & 0.639 & 0.473 & 0.269 \\
\hline \multirow{2}{*}{ SDRR } & $r$ & -0.095 & 0.181 & 0.185 & 0.382 & -0.194 & 0.15 & 0.098 & 0.225 & 0.098 & 0.255 & 0.013 & 0.235 \\
\hline & $\mathrm{p}$-value & 0.7 & 0.459 & 0.492 & 0.144 & 0.471 & 0.579 & 0.689 & 0.355 & 0.699 & 0.307 & 0.958 & 0.363 \\
\hline \multirow{2}{*}{ RMSSD } & $\mathrm{r}$ & -0.025 & 0.253 & 0.265 & 0.232 & -0.035 & 0.021 & 0.037 & 0.233 & 0.02 & 0.255 & -0.044 & 0.275 \\
\hline & p-value & 0.92 & 0.297 & 0.322 & 0.387 & 0.897 & 0.94 & 0.881 & 0.336 & 0.938 & 0.307 & 0.861 & 0.286 \\
\hline \multirow{2}{*}{ CVRR } & $\mathrm{r}$ & -0.295 & 0.035 & 0.347 & 0.456 & -0.144 & 0.138 & 0.189 & 0.267 & 0.129 & 0.115 & 0.018 & 0.074 \\
\hline & p-value & 0.22 & 0.887 & 0.188 & 0.076 & 0.594 & 0.61 & 0.437 & 0.27 & 0.61 & 0.651 & 0.945 & 0.779 \\
\hline \multirow{2}{*}{ VLF } & $\mathrm{r}$ & -0.219 & 0.247 & 0.182 & 0.479 & -0.344 & 0.212 & -0.032 & 0.382 & 0.307 & 0.094 & 0.055 & 0.414 \\
\hline & p-value & 0.367 & 0.307 & 0.499 & 0.06 & 0.192 & 0.431 & 0.898 & 0.106 & 0.216 & 0.711 & 0.829 & 0.098 \\
\hline \multirow{2}{*}{ LF } & $\mathrm{r}$ & -0.016 & 0.033 & 0.065 & 0.2 & -0.024 & 0.291 & -0.037 & 0.461 & 0.245 & 0.317 & 0.16 & 0.14 \\
\hline & p-value & 0.949 & 0.892 & 0.812 & 0.458 & 0.931 & 0.274 & 0.881 & $0.047 *$ & 0.328 & 0.2 & 0.526 & 0.593 \\
\hline \multirow{2}{*}{$\mathrm{HF}$} & $\mathrm{r}$ & -0.196 & -0.053 & 0.259 & 0.171 & 0.088 & 0.082 & 0.384 & 0.302 & 0.03 & 0.249 & 0.015 & 0.377 \\
\hline & p-value & 0.42 & 0.831 & 0.333 & 0.528 & 0.745 & 0.762 & 0.104 & 0.209 & 0.906 & 0.32 & 0.951 & 0.135 \\
\hline \multirow{2}{*}{$\begin{array}{l}\text { LF/HF } \\
\text { Ratio }\end{array}$} & $\mathrm{r}$ & 0.258 & 0.193 & -0.191 & 0.015 & -0.068 & 0.007 & -0.496 & -0.221 & 0.16 & -0.061 & 0.183 & -0.135 \\
\hline & $\mathrm{p}$-value & 0.286 & 0.429 & 0.478 & 0.957 & 0.803 & 0.978 & 0.031* & 0.363 & 0.526 & 0.81 & 0.468 & 0.606 \\
\hline \multirow{2}{*}{ SD1 } & $r$ & -0.025 & 0.253 & 0.265 & 0.232 & -0.035 & 0.021 & 0.037 & 0.233 & 0.02 & 0.255 & -0.044 & 0.275 \\
\hline & p-value & 0.92 & 0.297 & 0.322 & 0.387 & 0.897 & 0.94 & 0.881 & 0.336 & 0.938 & 0.307 & 0.861 & 0.286 \\
\hline \multirow{2}{*}{$\mathrm{SD} 2$} & $\mathrm{r}$ & -0.116 & 0.074 & 0.156 & 0.371 & -0.174 & 0.138 & 0.054 & 0.26 & 0.3 & 0.152 & 0.022 & -0.044 \\
\hline & p-value & 0.637 & 0.764 & 0.564 & 0.158 & 0.52 & 0.61 & 0.825 & 0.283 & 0.226 & 0.548 & 0.932 & 0.866 \\
\hline
\end{tabular}

LF parameter shows a positive number and more than 0.5 indicates that there is a strong direct relationship between the LF parameter and BP. The correlation between BP and HRV Poincaré Plot was found in the stroke group, with the p-value and correlation coefficient on the SD1 parameter $(0.02 \pm$
0.542) in the StandEC position. It was shown positive correlation between BP and SD1, which means that both of them have a direct association.

Based on several correlation tests that have been conducted between $\mathrm{BP}$ and $\mathrm{HRV}$, the correlation is only 
shown in the stroke group. A strong correlation is seen in the parameters SDRR, RMSSD, CVRR, LF, and SD1. The control group did not show any relationship between BP and HRV.

\subsection{Blood Flow Velocity and Heart Rate Variability}

The results of the BFV-HRV correlation test in the control group are shown in Table 10. The results in the table show the p-value $>0.05$ in all positions. This means that none of the HRV parameters in the group indicate a relationship with BFV.

Different results are shown in the stroke group. Correlation tests conducted in the stroke group showed a relationship between BFV and HRV in several positions.

Heart Rate Variability parameters that indicate a relationship with $\mathrm{BFV}$ are only found in the frequency domain HRV (p-value < 0.05). HRV parameters in the frequency domain that show a relationship between HRV and BFV are LF parameters and LF/HF Ratio. This relationship is only shown in the baseline and tilt positions

\section{Discussions}

This study compared Blood Pressure (BP), Blood Flow Velocity (BFV), and Heart Rate Variability (HRV) between control group and stroke group. The results of this study indicate that BP in the stroke group is higher than the control group in all positions. Hypertension is still become the most common risk factors in stroke patients [21] [22]. Other stroke mechanisms related to hypertension caused by mental stress [23] and increased sympathetic activity [24].

Changes in position also affect the results of BP measurement. BP was seen increasing between the two groups, as positions changed. At the baseline position, BP shows lower result than when sitting position [25]. Although BP in the stroke group was higher, it does not mean that stroke has caused BP increasing. In some cases, hypertension which then becomes a risk factor for stroke [26]. But one thing that is certain is that the average BP in the stroke group was indeed higher compared to the control group.

Blood Flow Velocity among right and left sides in stroke and control group has confirmed there is no significant difference in all positions. Although it was not showing a significant difference between the two groups, BFV in stroke group was slower than the control group in all positions. Blood Flow Velocity is influenced by blood vessel diameter, blood vessel elasticity, blood viscosity, and strength of heart muscle contraction. [1]

Deceleration BFV in the stroke group is possible due to blood viscosity. Blood viscosity has an influence on blood pressure [27]. Systolic blood pressure, diastole, and Mean Arterial Pressure (MAP) increase with increasing blood vascularity. In the subject demographics, there was no significant difference in the systole between the two groups. However, the mean diastole in the stroke group was significantly higher than the control group. Because blood pressure is influenced by systole and diastole, the high diastole value certainly increases MAP value. In addition, the increase in MAP is also due to the high viscosity. High viscosity influences slower blood flow in the blood vessels to the brain. The slowing down of $\mathrm{BFV}$ is then a risk factor for stroke [28].

Subject demographics showed significant differences between the control group and the stroke group on Body Mass Index (BMI). Nonetheless, research [20] [29] shows that BMI does not affect heart rate variability in obese subjects ( $p$-value > 0.05). Considering obesity is a risk factor for stroke, it can be concluded that the value of each HRV parameter is not absolutely caused by differences in BMI.

In common, HRV in the stroke group was lower than in the control group. Some similar medical conditions are also found in certain diseases. Elderly hypertensive patients have decreased heart rate variability [30]. Significant decreases in HRV are also found in people with psychiatric disorders such as schizophrenia [31] and bipolar disorder [32].

The decrease in HRV in the stroke group indicates that there is interference in the autonomic nervous system. The lower variability in the stroke group is made possible by obstruction of the hemisphere and medullary rods which then damage the autonomic system, resulting abnormalities in the HRV characteristics [33]. The decrease in parasympathetic activity in the stroke group is shown by the low value of the RMSSD and SD1 parameters. Although the RMSSD and SD1 parameters between the two groups in this study did not show any significant differences in various positions, the differences were significantly seen through the HF parameters at the baseline position.

Previous studies have shown that RMSSD is associated with HF in men during sleep [34]. The relationship between the two parameters shows indirectly differences in parasympathetic system activity between the stroke group and the control group. It can be seen in the baseline position through the HF parameter. During breathing, the cardiorespiratory center blocks the flow of vagal outflow, causing the heart beating faster [35]. A faster heart rate indicates a high frequency (HF) in the frequency domain.

Sympathetic and parasympathetic nervous system activities contribute to the SDRR parameters [36]. Research conducted by [8] found that low SDNN in patients with sepsis can be an important predictor related to patient mortality in hospitals with a prediction rate of $70 \%$. In general, SDRR parameters in the control group were higher than the stroke group. These conditions indicate that the autonomic nervous system in the control group is much better. Significant differences in the SDRR parameters can only be seen in the baseline and StandEO positions. Disregulation of the control of the autonomic nervous system is associated with high sympathetic nerves and decreased parasympathetic nerves [37].

RMSSD parameters as a representation of parasympathetic activity showed no significant differences in the two groups. SDRR which is a representation of the sympathetic and parasympathetic nervous system, showed a significant difference between the stroke group and the control group. If the parasympathetic nervous system does not show any difference, then a significant difference in the SDRR must be due to sympathetic nervous system activity. 
Several studies have shown an association between SDRR parameters with LF and VLF parameters [38] [39] [40]. The same result is shown by the significant difference between the SDRR parameters and the LF parameters at the baseline position, and the SDRR parameters with VLF at the StandEO position. Other studies [41] [42] [43] also mentioned that the high activity of the sympathetic nervous system in the frequency domain is reflected through the low LF parameters. Similar results were also found in [44], which showed that the mean LF in 329 fibromyalgia patients $(388.99 \mathrm{~Hz})$ was significantly lower compared to 58 normal patients $(470.0 \mathrm{~Hz})$.

The control group had higher mean VLF and LF than the stroke group. The average VLF and LF in the control group were $344.93 \mathrm{~s}^{2} / \mathrm{Hz}$ and $206.64 \mathrm{~s}^{2} / \mathrm{Hz}$, whereas in the stroke group were $165.68 \mathrm{~s}^{2} / \mathrm{Hz}$ and $103.37 \mathrm{~s}^{2} / \mathrm{Hz}$. Under normal conditions, healthy subjects do show an increase in VLF at rest [45]. The same condition was also shown in this study, where the stroke group had a lower mean VLF and LF compared to the control group. This means that there is a parasympathetic nervous system disorder, which in this case is characterized by high sympathetic activity in the stroke group. Low VLF at resting conditions is associated with increased chronic inflammation [46] and death from arrhythmias [47]. Excessive sympathetic nerve activity is also shown in people with hypertension [48]. Several factors cause high sympathetic activity, including genetic factors, stress, obesity, and absence of physical activity [49]. In people with stroke, these factors can then increase sympathetic nerve activity.

Autonomic nervous system activity can also be seen through the parameters SD1 and SD2 found in non-linear analysis using Poincaré Plot. SD1 (short-term variability) parameters related to parasympathetic nervous system activity in the stroke group showed a lower mean. As explained earlier, although the stroke group had a lower SD1, no significant difference was found between the two groups. The parameter SD2 (long-term variability) in the Poincaré Plot shows more activity from the sympathetic nervous system. The lower SD2 parameter indicates that the sympathetic nervous system activity is getting higher [50] [51]. The stroke group had a lower SD2 value compared to the control group. The most significant difference was seen in the baseline and StandEO positions. This is consistent with the results of the analysis using a linear method, that increased sympathetic nervous system activity is also seen through Poincaré Plot analysis on SD2 parameters. The results of this study at the same time confirm previous studies [52], that ischemic stroke is largely characterized by the dominance of sympathetic nervous system activity.

Total function of the autonomic nervous system is shown by using the CVRR parameter, while the change in autonomic nerve activity from sympathetic to parasympathetic is indicated through the LF/HF Ratio. The CVRR parameter in the stroke group lower than the control group. Significant differences between the two groups were only shown in the baseline position. Thus, the total function of the autonomic nervous system in the control group is better than the stroke group, especially seen at the baseline position. Significant differences between the two groups were not seen in changes in autonomic nerve activity from sympathetic to parasympathetic, which in this case was represented through the $\mathrm{LF} / \mathrm{HF}$ Ratio. Similar results were also found in studies [53] [54].

Regarding BP and BFV in the control and stroke group, the results of this study showed that in the control group, there was a strong relationship at the SitEC position. The negative correlation between $\mathrm{BP}$ and $\mathrm{BFV}$ in the control group shows that the higher BP, will be followed by lower BFV. The mechanism of CA in healthy subjects involves rapid adjustment of brain blood vessels, so that $\mathrm{BFV}$ remains stable in response to changes in BP [8]. In the stroke group, there was no relationship between BP and BFV. Thus, BP and BFV, which affect the CA mechanism, have indeed been reduced after a stroke [8] [9].

In the control group no relationship was found between $\mathrm{BP}$ and HRV in all domains. Changes in position and physical activity do allow changes in HRV, but do not have a significant effect on BP [55]. Different results were found in the stroke group. A significant relationship was found between BP and HRV (SDRR, RMSSD, CVRR, LF, SD1). Sympathetic activation contributes to increasing blood pressure in hypertensive patients. This is what then causes high blood pressure in the stroke group. The positive correlation between HRV and BP in the stroke group indicates that higher sympathetic nerve activity, causing BP to increase.

There was no relationship between BFV and HRV in the control group, whereas in the stroke group this relationship was seen in the LF parameter and the LF/HF Ratio. BFV in the MCA will increase according to increasing heartbeat [56]. Improving the heartbeat is a form of sympathetic nervous system. A positive correlation between sympathetic nerve (LF) and BFV is also shown in stroke patients. Stroke sufferers who are more dominated by sympathetic nerve activity show an increase in the speed of blood flow to meet the blood supply to the brain. This also confirms the research [57], that the CA mechanism related to BFV in MCA can operate efficiently in the LF range rather than the HF range.

The correlation shown between BFV and HRV in this study is not strong enough to show the overall CA mechanism. The CA mechanism in stroke patients which is very complex and involves many other regulations (such as myogenic, neurogenic, metabolic, etc.) need a further research.

\section{CONCLUSION}

Blood pressure (BP) in the ischemic stroke group with some exclusions in this study was higher than in the control group, whereas Blood Flow Velocity (BFV) in the ischemic stroke group was faster than the control group. Almost all parameters in the Heart Rate Variability (HRV) in the stroke group were lower than the control group.

In the control group, BP is inversely proportional to BFV ( $\mathrm{p}$-value $<0.05$ and $\mathrm{r} \geq 0.5$ ). In patients with ischemic stroke, these changes cannot be done properly, so no difference is found between BP and BFV. There is a relationship between $\mathrm{BP}$ and HRV in the ischemic stroke group, which is 
associated with high sympathetic nerve activity (p-values < 0.05 and $r>0.4$ ). The relationship between BFV and HRV was also only found in the stroke group (p-value $<0.05$ and $r>0.4$ ).

Parameters on HRV that can be used to distinguish stroke characteristics are SDRR, CVRR, LF, and VLF which describe sympathetic and parasympathetic activities, and RMSSD and SD1 which show parasympathetic nerve activity.

Subject quantity in further studies can be added, as well as ischemic stroke and control subject groups can be categorized based on age and gender. Biosignal used to determine the condition of ischemic stroke patients can be combined with other biosignals. It is possible to analyze the mechanism of CA in stroke patients by using myogenic and metabolic regulation.

\section{ACKNOWLEDGMENTS}

We thank to the all members of Medical Biomedical Research Unit in Islamic University of Indonesia.

\section{REFERENCES}

[1] C. Molnar and J. Gair, "Blood Flow and Blood Pressure Regulation," in Concepts of Biology-1st Canadian Edition, Texas, OpenStax College, 2013, pp. 25-30.

[2] E. J. Benjamin, S. S. Virani, C. W. Callaway, A. M. Chamberlain, A. R. Chang and S. Cheng, "Heart Disease and Stroke Statistics-2018 Update," American Heart Association, Dallas, 2018.

[3] M. Lutski, I. Zucker, T. Shohat and D. Tanne, "Characteristics and Outcomes of Young Patients with First-Ever Ischemic Stroke Compared to Older Patients: The National Acute Stroke ISraeli Registry," Front Neurol., vol. 8, p. 421, 2017.

[4] K. Nedeltchev, T. A. der Maur, D. Georgiadis, M. Arnold, V. Caso, H. P. Mattle, G. Schroth, L. Remonda, M. Sturzenegger, U. Fischer and R. W. Baumgartner, "Ischaemic stroke in young adults: predictors of outcome and recurrence," J. Neurol Neurosurg Psychiatry, vol. 76, no. 2, pp. 191-195, 2005.

[5] A. Adunsky, M. Hershkowitz, R. Rabbi, L. Asher-Sivron and A. Ohry, "Functional recovery in young stroke patients," Arch. Phys. Med. Rehabil, vol. 73, no. 9, pp. 859-862, 1992.

[6] D. Smajlovic, D. Salihovic, O. C. Ibrahimagic and O. Sinanovic, "Characteristics of stroke in young adults in Tuzla Canton, Bosnia and Herzegovina," Coll Antropol, vol. 37, no. 2, pp. 515-519, 2013.

[7] M. A. Ritter, A. Rohde, P. U. Heuschmann, R. Dziewas, J. Stypmann, D. G. Nabavi and B. E. Ringelstein, "Heart rate monitoring on the stroke unit. What does heart beat tell about prognosis? An observational study," BMC Neurology, vol. 11, no. 47, pp. 1-8, 2011.

[8] Z. Chen, K. Hu, H. E. Stanley, V. Novak and P. C. Ivanov, "Crosscorrelation of instantaneous phase increments in pressureflow fluctuations: Applications to cerebral autoregulation," Physical review. E, Statistical, nonlinear, and soft matter physics, vol. 73, p. 031915, 2006.

[9] M. J. Aries, J. W. Elting, J. D. Keyser, B. P. Kremer and P. C. Vroomen, "Cerebral Autoregulation in Stroke," Stroke, vol. 41, pp. 2697-2704, 2010.

[10] J. P. Appleton, N. Sprigg and P. M. Bath, "Blood pressure management in acute stroke," Stroke and Vascular Neurology, vol. 1, no. 2, pp. 7282, 2016.

[11] M. C. Aoi, K. Hu, M.-T. Lo, M. Selim, M. S. Olufsen and V. Novak, "Impaired Cerebral Autoregulation Is Associated with Brain Atrophy and Worse Functional Status in Chronic Ischemic Stroke," PLoS ONE, vol. 7, no. 10, p. e46794, 2012.

[12] G. Ernst, "Heart-Rate variability - More than Heart Beats?," Front. Public Health, vol. 5, no. 240, 2017.

[13] V. Novak, "Cerebral Vasoregulation in Elderly with Stroke," MIT Laboratory for Computational Physiology, 4 October 2018. [Online].
Available: https://physionet.org/content/cves/1.0.0/. [Accessed 26 March 2019].

[14] V. Novak, K. Hu, L. Desrochers, P. Novak, L. Caplan, L. Lipsitz and M. Selim, "Cerebral flow velocities during daily activities depend on blood pressure in patients with chronic ischemic infarctions," Stroke: a Journal of Cerebral Circulation, vol. 41, no. 1, pp. 61-66, 2010.

[15] E. O'Brien, R. Asmar, L. Beilin, Y. Imai, J. Mallion, G. Mancia, T. Mengden, M. Myers, P. Padfield, P. Palatini, G. Parati, T. Pickering, J. Redon, J. Staessen, G. Stergiou and P. Verdecchia, "European Society of Hypertension recommendations for conventional, ambulatory and home blood pressure measurement," J. Hypertens., vol. 21, no. 5, pp. 821-848, 2003.

[16] P. Eames, M. Blake, S. Dawson, R. Panerai and J. Potter, "Dynamic cerebral autoregulation and beat-to-beat blood pressure control are impaired in acute ischaemic stroke.," J. Neurol Neurosurg Psychiatry, vol. 72, pp. 467-473, 2002.

[17] S. Schwarz, A. Georgiades, A. Aschoff and S. S., "Effects of induced hypertension on inctracranial pressure and flow velocities of the middle cerebral arteries in patients with large hemispheric stroke," Stroke, vol. 33, pp. 998-1004, 2002.

[18] V. Novak, A. Chowdhary, B. Farrar, H. Nagaraja, J. Braun, R. Kanard, P. Novak and A. Slivka, "Altered cerebral vasoregulation in hypertension and stroke," Neurology, vol. 60, pp. 1657-1663, 2003.

[19]N. Smalheiser, Data Literacy 1st Edition How to Make Your Experiments Robust and Reproducible, Illinois, USA: Academic Press, 2017.

[20] R. L. Yadav, P. K. Yadav, L. K. Yadav, K. Agrawal, S. K. Sah and M. N. Islam, "Association between obesity and heart rate variability indices: an intuition toward cardiac autonomic alteration - a risk of CVD," Diabetes Metab. Syndr. Obes., vol. 10, pp. 57-64, 2017.

[21] R. Bowry, D. D. Navalkele and N. R. Gonzales, "Blood pressure management in stroke: Five new things," Neurology Clinical Practice, vol. 4, no. 5, pp. 419-426, 2014.

[22]Z. Gaciong, M. Siński and J. Lewandowski, "Blood Pressure Control and Primary Prevention of Stroke: Summary of the Recent Clinical Trial Data and Meta-Analyses," Current Hypertension Reports, vol. 15, no. 6, pp. 559-574, 2013.

[23]B. Carlberg, K. Asplund and E. Hagg, "Course of blood pressure in different subsets of patients after an acute stroke," Cerebrovasc Dis, vol. 1, no. 5, pp. 281-287, 1991.

[24]P. Jansen, B. Schulte, E. Poels and F. Gribnau, "Course of blood pressure after cerebral infarction and transient ischemic attack," Clin Neurol Neurosurg, vol. 89, no. 4, pp. 243-246, 1987.

[25] T. G. Pickering, J. E. Hall, L. J. Appel, B. E. Falkner, J. Graves, M. N. Hill, D. W. Jones, T. Kurtz, S. G. Sheps and E. J. Roccella, "Recommendations for Blood Pressure Measurement in Humans and Experimental Animals," Hypertension, vol. 45, no. 1, pp. 142-161, 2005.

[26] S. Ishikawa, K. Kazuomi, K. Kayaba, T. Gotoh, N. Nago, Y. Nakamura, A. Tsutsumi and E. Kajii, "Linear relationship between blood pressure and stroke: the Jichi Medical School Cohort Study," J. Clin. Hypertens (Greenwich), vol. 9, no. 9, pp. 677-683, 2007.

[27] T. Gori, P. S. Wild, R. Schnabel, A. Schulz, N. Pfeiffer, M. Blettner, M. E. Beutel, S. Forconi, F. Jung, K. J. Lackner, S. Blankenberg and T. Münzel, "The distribution of whole blood viscosity, its determinants and relationship with arterial blood pressure in the community: crosssectional analysis from the Gutenberg Health Study," Therapeutic Advances in Cardiovascular Disease, pp. 354-365, 2015.

[28] S. H. Song, J. H. Kim, J. H. Lee, Y.-M. Yun, D.-H. Choi and H. Y. Kim, "Elevated blood viscosity is associated with cerebral small vessel disease in patients with acute ischemic stroke," BMC Neurol, vol. 17, no. 1, p. 20, 2017.

[29] I. Antelmi, R. S. D. Paula, A. R. Shinzato, C. A. Peres, A. J. Mansur and C. J. Grupi, "Influence of Age, Gender, Body Mass Index, and Functional Capacity on Heart Rate Variability in a Cohort of Subjects Without Heart Disease," Am. J. Cardiol., vol. 93, pp. 381-385, 2004.

[30]P. E. d. Andrade, J. A. T. d. Amaral, L. d. S. Paiva, F. Adami, J. Z. Raimudo, V. E. Valenti, L. C. d. Abreu and R. D. Raimundo, "Reduction of heart rate variability in hypertensive elderly," BLOOD PRESSURE, 2017.

[31]E. Moon, S.-H. Lee, D.-H. Kim and B. Hwang, "Comparative Study of Heart Rate Variability in Patients with Schizophrenia, Bipolar Disorder, Post-traumatic Stress Disorder, or Major Depressive 
Disorder," Clin. Psychopharmacol Neurosci, vol. 11, no. 3, pp. 137143, 2013

[32]O. Carr, M. d. Vos and K. E. A. Saunders, "Heart rate variability in bipolar disorder and borderline personality disorder: a clinical review," Evid. Based Ment. Health, vol. 21, no. 1, pp. 23-30, 2018.

[33]J. Korpelainen, K. Sotaniemi, A. Mäkikallio, H. Huikuri and V. Myllylä, "Dynamic behavior of heart rate in ischemic stroke," Stroke, vol. 30, no. 5, pp. 1008-1013, 1999.

[34]H. Otzenberger, C. Gronfier, C. Simon, A. Charloux, J. Ehrhart, F. Piquard and G. Brandenberger, "Dynamic heart rate variability: a tool for exploring sympathovagal balance continuously during sleep in men," Am. J. Physiol., vol. 275, no. 3, pp. 946-950, 1998.

[35]D. Eckberg, "Human sinus arrhythmia as an index of vagal outflow," Journal of Applied Physiology, pp. 961-966, 1983.

[36]F. Shaffer and J. P. Ginsberg, "An Overview of Heart Rate Variability Metrics and Norms," Frontiers in Public Health, vol. 5, p. 258, 2017.

[37]J. Sztajzel, "Heart rate variability: a noninvasive electrocardiographic method to measure the autonomic nervous system," Swiss Med Wkly, vol. 134, pp. 514-522, 2004

[38] J. J. Bigger, J. Fleiss, R. Steinman, L. Rolnitzky, R. Kleiger and J. Rottman, "Correlations among time and frequency domain measures of heart period variability two weeks after acute myocardial infarction," Am. J. Cardiol., vol. 69, no. 9, pp. 891-898, 1992

[39] K. Umetani, D. Singer, R. McCraty and M. Atkinson, "Twenty-four hour time domain heart rate variability and heart rate: relations to age and gender over nine decades," J. Am. Coll. Cardiol., vol. 31, no. 3, pp. 593-601, 1998.

[40]C. Calvert and T. Wall, "Correlations among time and frequency measures of heart rate variability recorded by use of a Holter monitor in overtly healthy Doberman pinschers with and withou echocardiographic evidence of dilated cardiomyopathy," Am. J. Vet. Res., vol. 62, no. 11, pp. 1787-1792, 2001

[41]D. Lucini, G. Norbiato, M. Clerici and M. Pagani, "Hemodynamic and autonomic adjustments to real life stress conditions in humans," Hypertension, vol. 39, no. 1, pp. 184-188, 2002.

[42] J. Brosschot, E. van Dijk and J. Thayer, "Daily worry is related to low heart rate variability during waking and the subsequent nocturnal sleep period," Int. J. Psychophysiol, vol. 63, no. 1, pp. 39-47, 2007.

[43]F. Lombardi and P. Stein, "Origin of heart rate variability and turbulence: An appraisal of autonomic modulation of cardiovascular function," Front. Physiol., vol. 2, no. 95, 2011.

[44]D. S. Silver, S. R. Markoff, L. Naghi, M. Silver and L. May, "Reduction in Parasympathetic Autonomic Nervous System Function in Fibromyalgia Patients," Arch Med, vol. 8, pp. 1-7, 2016.

[45] R. McCraty, Science of the Heart, Volume 2 Exploring the Role of the Heart in Human Performance An Overview of Research Conducted by the HeartMath Institute, 2016.
[46] R. Lampert, J. Bremner, S. Su, A. Miller, F. Lee, F. Cheema, J. Goldberg and V. Vaccarino, "Decreased heart rate variability is associated with higher levels of inflammation in middle-aged men," Am Heart J., vol. 156, no. 4, pp. 759.e1-7, 2008.

[47]J. J. Bigger, J. Fleiss, R. Steinman, L. Rolnitzky, R. Kleiger and J. Rottman, "Frequency domain measures of heart period variability and mortality after myocardial infarction," Circulation, vol. 85, no. 1, pp. 164-171, 1992.

[48]N. Verma, "Sympathetic Nervous System and Hypertension," Hypertension Journal, vol. 3, no. 1, pp. 27-36, 2017.

[49]M. Esler, "The Sympathetic System and Hypertension," American Journal of Hypertension, vol. 13, no. 3, pp. 99S-105S, 2000.

[50]P. Kamen, H. Krum and A. Tonkin, "Poincaré plots of heart rate variability allows quantitative display of parasympathetic nervous activity in humans," Clinical Science, vol. 91, no. 2, pp. 201-208, 1996.

[51]M. Tulppo, T. Mäkikallio, T. Takala, T. Seppänen and H. Huikuri, "Quantitative beat-to-beat analysis of heart rate dynamics during exercise," American Journal of Physiology, vol. 271, no. 1, pp. H244252, 1996.

[52]L. Guan, J.-P. Collet, G. Mazowita and V. E. Claydon, "Autonomic Nervous System and Stress to Predict Secondary Ischemic Events after Transient Ischemic Attack or Minor Stroke: Possible Implications of Heart Rate Variability," Front Neurol, vol. 9, p. 90, 2018.

[53]E.-S. A. Shokr, "Effect of Exam Stress on Heart Rate Variability Parameters in Healthy Students," Egypt. Acad. J. Biol. Sci., vol. 7, no. 1, pp. 75-81, 2015 .

[54] S. Hammoud, R. Karam, R. Mourad, I. Saad and M. Kurdi, "Stress and Heart Rate Variability during University Final Examination among Lebanese Students," Behav. Sci., vol. 9, no. 1, p. 3, 2019.

[55] M. E. Papp, P. Lindfors, N. Storck and P. E. Wändell, "Increased heart rate variability but no effect on blood pressure from 8 weeks of hatha yoga - a pilot study," BMC Research Notes, vol. 6, no. 59, 2015.

[56]N. Wahlgren, G. Hellström, C. Lindquist and A. Rudehill, "Sympathetic Nerve Stimulation in Humans Increases Middle Cerebral Artery Blood Flow Velocity," Cerebrovasc Dis, vol. 2, no. 6, pp. 359364, 1992.

[57]T. B.-J. Kuo, C.-M. Chern, W.-Y. Sheng, W.-J. Wong and H.-H. Hu, "Frequency Domain Analysis of Cerebral Blood Flow Velocity and its Correlation with Arterial Blood Pressure," Journal of Cerebral Blood Flow \& Metabolism, vol. 18, no. 3, pp. 311-318, 1998 